\title{
Declining Working Phone Rates Impact Sample Efficiency
}

Linda B Piekarski

Tags: survey practice

DOI: $10.29115 /$ SP-2009-0032

\section{Survey Practice}

Vol. 2, Issue 7, 2009

Declining Working Phone Rates Impact Sample Efficiency

Working Phone Rate (WPR) usually means the proportion of telephone numbers in a telephone sample that connects to a residence and the WPR for landline telephone samples has been steadily declining since the early 1990's. This decline has affected phone room efficiencies, interviewer morale and ultimately survey costs. This paper addresses the various causes for the decline and what they may mean for the future of telephone surveying.

The WPR of a sample can be adversely affected by a number of factors, singly or in combination: sample design, sample geography, listed rates, population density, mobility rates, number of businesses, telephone numbering plan changes, number demand, number assignment policies, advances in telephony and the length of time between sample selection and the fielding of the sample.

In survey research, WPR really refers to the proportion of telephone numbers in a sample that are working numbers. Non-working numbers include numbers that are not-in-service, disconnected or have been changed. However, a working number may not be an eligible number. Ineligible units would be those numbers that connect to a unit such as a business, a modem or a fax machine. A more precise term is Working Residential Rate (WRR) or the proportion of telephone numbers that connects to a residence.

Table 1 demonstrates the correct way to calculate the WRR for a sample. It is important to note that $\boldsymbol{B}$ is the number of sample records dialed not the number of dialing attempts. 
Table 1 Calculating Working Residential Phone Rate.

$\begin{array}{lll}\text { Dialing Outcome } & \text { WFR } \\ \text { Disconnected } & 500 & \\ \text { Business/Government } & 80 & \\ \text { Faxes } & 5 & \\ \text { Modems } & 5 & \\ \text { Non Residential (A) } & 590 & 59 \% \\ \text { Total Dialed (B) } & 1000 & 41 \% \\ \text { A divided by B } & & \end{array}$

Since non-working numbers are easily identified as out-of-scope early in the interview process, they do not add much to overall survey costs. Unidentified business numbers however are sometimes more difficult to pre-identify or identify on the first dialing attempt, without a daytime call attempt, and their presence may have a modest impact on cost.

Sample providers can identify and remove many business numbers. However, businesses today have multiple voice lines (rollover lines, Direct Inward Dial lines, fax lines, and modem lines) which are often not available on commercial business files and cannot always be pre-identified. As the number of out-of-scope numbers in a sample increases, the amount of sample required to complete the target number of interviews also increases.

In the above example, a WRR of 41 percent requires a sample size of 1220 to yield 500 working residential numbers. If the WRR drops to 30 percent then a sample size of 1667 will be needed to achieve the same number of working residential numbers. The increase in sample required to complete a survey does not add much to survey costs, but the additional phone room effort required to resolve them can.

The WRR will vary according to sampling methodology. A sample of directory-listed telephone numbers will usually have a higher WRR than a Random Digit Dial (RDD) sample, but will be less representative. Conversely, a RDD sample will have a lower WRR than a directory-listed sample but be more representative.

Efficiency gains for RDD samples can be achieved by using non-epsem sampling methodologies. Samples can be selected from a frame that has been truncated based on the presence or number of listed phones in a 100-block or 1000-block. Selecting a sample from a frame of 100-blocks with three or more listed numbers or five or more listed numbers will increase efficiency but at the expense of representation.

Probability Proportionate to Size (PPS) RDD samples can also achieve higher working residential rates. PPS RDD samples are selected using the number of listed phones in each 100-block as a Measure of Size (MOS) during sample 
selection. Such samples will be proportionate to listed numbers while still including non-listed numbers but must be weighted to account for disproportionate sampling.

\section{AVERAGE WRR BY SAMPLE TYPE:}

Directory-Listed - 80\%

PPS RDD - $44 \%$ (range $34 \%$ to $59 \%$ by state)

Epsem - 31\% (range 22\% to $43 \%$ by state)

For directory-listed samples, the WRR is primarily affected by the mobility of the population. Since an average of 18 percent of households move in a year, 18 percent of directory-listed numbers might have been disconnected by the time a sample is selected in an area where a new directory is about to be published.

Higher mobility rates in urban areas can result in higher disconnect rates for listed samples from urban areas. Conversely, lower mobility rates in rural areas can mean lower disconnect rates. However, directory-listed samples will under-represent new listings, Voice over Internet Protocol (VoIP) and cable telephone numbers which are often unpublished.

For RDD samples, the WRR will be lower than for directory-listed samples. RDD samples improve the coverage of telephone households by including numbers that are not found in directories or otherwise obtained by compilers. However, many of the not-listed RDD numbers will be either non-working or ineligible resulting in a lower WRR.

The WRR for an RDD sample is directly proportional to the number of residences present in the frame of possible RDD numbers. Over the decade of the 1990's list-assisted RDD frames grew dramatically as the demand for telephone numbers skyrocketed. Since then, the demand for residential telephone numbers has been steadily declining. Multiple residential lines are being replaced by DSL, VoIP or cable connections and more and more households have been substituting wireless phones for their landline phones. Table 2 shows how these changes have affected the list-assisted telephone frame. The estimated 87 million landline households are now scattered across over 282 million possible numbers. 
Table 2 Telephone Frame Coverage of Households.

\begin{tabular}{lllll}
\hline & & \% Households & \% Telephone Households & \% Landline Households \\
Total 2008 US Households & $113,138,520$ & & & \\
Esc. Telephone Households & $111,020,044$ & $97.7 \%$ & $21.7 \%$ & $78.3 \%$ \\
Est. Cell Only Households & $24,090,522$ & $21.2 \%$ & $58.2 \%$ & $74.3 \%$ \\
Est. Landline Households & $86,930,422$ & $76.5 \%$ & & $30.8 \%$ \\
Directory Listed Households & $64,625,185$ & $56.9 \%$ & & \\
List-Assisted RDD Frame & $282,271,600$ & & & \\
Epsem WRR & & &
\end{tabular}

Sources: Households: ScanUS 2008; Telephone \& Listed households: Survey Sampling Intl. 2009; Cell-only households: Mediamark Research 2009.

The demise of mechanical switching has more or less eliminated the clustering of residential numbers within 100-blocks. As a result, the number of listed, and by inference unlisted households, in a 100-block has steadily declined. While the percent of landline households that are directory-listed households has not changed much (74\%) over the past twenty years, there are simply more 100-blocks and fewer landline telephone households.

The average proportion of listed phones in a 100-block (and consequently in an epsem sample) has declined from 44 percent in 1986 to 23 percent in 2009. The median block size (reflecting the proportion of listed numbers in a PPS sample) has declined from 53 percent in 1986 to 31.0 percent in 2009.

Other factors can affect Working Residential Rates. Local Number Portability (LPN) is the ability of a subscriber to change his telephone service provider without changing his telephone number. The FCC reported that as of September 200857.2 million landline telephone numbers had been ported to another landline provider. Porting requires two 10-digit numbers, one for the original subscriber number and the other for the switch belonging to the new carrier. The resulting presence of 57 million non-connecting ports in the RDD frame has helped to contribute to the overall decline in WRR.

How local telephone companies assign telephone numbers within their allotted pool of numbers has also affected WRR. In an effort to conserve telephone numbers, 1000-blocks pooling was mandated by the FCC. This conservation measure declared that service providers could no longer monopolize all 10,000 in a prefix. They are required to return unused 1000-blocks to a pool administrator for reassignment to other service providers.

The plan has had its desired effect. The Federal Communication Commission (FCC) reported that as of June 30, 2008, only 48.1 percent of available numbers have been assigned to end users ( $58 \%$ to residential subscribers and $42 \%$ to business subscribers).

However according to FCC data for June 2008 in Table 3, number utilization 
varies by carrier and by geography.

Table 3 Number Utilization by Carrier Type.

$\begin{array}{llll}\text { Carrier Type } & \text { \% Assigned } & \text { \% in Non-Rural } & \% \text { in Rural } \\ \text { Incumbent Local Exchange Carriers (ILEC) } & 50.3 \% & 54.1 \% & 16.3 \% \\ \text { Competitive Local Exchange Carriers (CLEC) } & 30.4 \% & 31.0 \% & 8.1 \%\end{array}$

Competitive Local Exchange Carriers (CLEC) include cable and VoIP service providers. The substantially lower utilization rates for CLECs and for rural areas suggest that Working Residential Rates can be lower in rural areas and in prefixes belonging to CLECs.

\section{SUMMARY}

Wireless substitution, digital telephony and various non-residential uses of telephone numbers will continue to erode the incidence of residential landline telephone numbers in the wireline frame. Using alternative methodologies such as listed frames, truncated frames or PPS sampling can improve Working Residential Rates but at the expense of coverage and representation. In order to address the wireless substitution trend, cell phone surveys are becoming a necessary component of all telephone surveys. Sampling companies will continue to explore other solutions, but such solutions will probably not get us back to the golden age of RDD efficiencies. 


\section{REFERENCES}

Federal Communications Commission. 2009a. "Local Telephone Competition: Status as of June 30, 2008." Washington, D.C. http://hraunfoss.fcc.gov/edocs_public/attachmatch/ DOC-292193A1.pdf.

- - 2009b. "Numbering Resource Utilization in the United States as of June 30, 2008." Washington, D.C. http://hraunfoss.fcc.gov/edocs_public/attachmatch/DOC-289173A1.pdf. 